\title{
Invasion of Muscularis Mucosa Present
}

National Cancer Institute

\section{Source}

National Cancer Institute. Invasion of Muscularis Mucosa Present. NCI Thesaurus. Code C96189.

A morphologic finding indicating the presence of a malignant cellular infiltrate invading the muscularis mucosa. 